\title{
El perfil actual del estudiante de pregrado de la Facultad de Estudios a Distancia de la UMNG y su preferencia al estudiar un posgrado*
}

\author{
Henry Acuña Barrantes ' \\ Martin Elías Villamil Rozo ${ }^{2}$ \\ Eduardo Padilla Beltrán ${ }^{3}$
}

Recibido: 23-12-2019

Aceptado: 03-05-2020

\section{Resumen}

En este artículo se busca actualizar el perfil de los estudiantes que cursan pregrados en modalidad a distancia-virtual, matriculados de la Facultad de Estudios a Distancia (Faedis) de la Universidad Militar Nueva Granada, haciendo una comparación entre los períodos 2015-II y 2018-II, cotejando sus aspectos económicos, demográficos e institucionales. Se concurrió a la metodología descriptiva realizando encuestas a una muestra del $14,2 \%$ de la

\footnotetext{
*Artículo de investigación relacionado con el proyecto institucional de la Faedis titulado "El perfil del estudiante de la Facultad de Estudios a Distancia y su expectativa", con vigencia de 2015-2018.

1. Doctor y Máster en Etica Global y Negocios Internacionales de la Escuela Española de Negocios Internacionales (EENI.) Maestria en Educacion de la Universidad Internacional de la Rioja (UNIR), Economista, especialista en Alta Gerencia de la Universidad Militar Nueva Granada (UMNG) y Docente de la (EENI). Integrante del Grupo Liderazgo de la (UMNG), clasificado A por Colciencias. Profesional Especializado de la Facultad de Estudios a Distancia.

Correo electrónico: henry.acuna@unimilitar.edu.co ORCID: https://orcid.org/0000-0001-5321-6401
}

2. Ingeniero Industrial; especialista en Aprendizaje Autónomo; magíster en Pedagogía, Universidad Santo Tomás. Profesor asociado, Programa de Ingeniería Industrial a Distancia, Universidad Militar Nueva Granada.

Correo electrónico: martin.villamil@unimilitar.edu.co

ORCID: https://orcid.org/0000-0003-4784-0739

3. Posdoctorado en Educación, Ciencias Sociales e Interculturalidad, Universidad Santo Tomás; Doctor of Education, Newport University. Docente asociado, director del Centro de Investigaciones, Facultad de Estudios a Distancia; líder del Grupo de Investigación Pedagogía y Didáctica en Educación Superior (PYDES), Universidad Militar Nueva Granada.

Correo electrónico: eduardo.padilla@unimilitar.edu.co

ORCID: https://orcid.org/0000-0003-4784-0739 
comunidad estudiantil activa. Se observó que la mayoría de los alumnos que respondieron la encuesta están en el rango de edad de 26 a 45 años, lo que demuestra que es una población adultojoven; sin embargo, el grupo de 17-20 años presenta un aumento importante. De igual forma, los estudiantes demandan prontamente que la Facultad oferte un mayor número de especializaciones y maestrías, y entre su interés está la Maestría en Alta Gerencia (MBA), y esperan que su enfoque sea hacia la profundización.

Palabras clave: educación superior virtual, modalidad a distancia, modalidad virtual, estudiante de pregrado, estudiante de posgrado.

\section{The current profile of the undergraduate student of the Faculty of Distance Studies of the UMNG and their preference when studying a postgraduate degree}

\section{Abstract}

This article seeks to update the profile of students who are studying undergraduate degrees in virtual-distance mode, enrolled in the Faculty of Distance Studies (Faedis) of the Nueva Granada Military University, making a comparison between the periods 2015-II and 2018 -II, comparing their economic, demographic and institutional aspects. The descriptive methodology was used by surveying a sample of $14.2 \%$ of the active student community. It was observed that most of the students who responded to the survey are in the age range of 26 to 45 years, which shows that it is an adult-young population; however, the 17-20 year old group shows a significant increase. Similarly, students promptly demand that the Faculty offer a greater number of specializations and master's degrees, and among their interest is the Master's in Senior Management (MBA), and they hope that their focus will be towards deepening. 
Keywords: virtual higher education, distance modality, virtual modality, undergraduate student, graduate student.

\section{Introducción}

En el año 2015 se realizó un estudio entre los estudiantes activos de la Universidad Militar Nueva Granada (UMNG) que se encontraban adscritos a la Facultad de Estudios a Distancia (Faedis). Con relación al incremento de la oferta en la modalidad virtual-a distancia en la educación superior a nivel nacional, se buscó hacer un seguimiento a una población de estudiantes de pregrado de la Faedis registrados durante la vigencia 2015-II. En esta investigación se buscó realizar una comparación de estos con un grupo de estudiantes matriculados en esta misma facultad durante el semestre el 2018-II, mediante un instrumento como lo es la encuesta, buscando con ella obtener datos en lo referente a aspectos económicos, demográficos e institucionales, con el fin de dar respuesta a la pregunta de investigación: ¿ha cambiado el perfil de los estudiantes de pregrado de la Facultad de Estudios a Distancia?

\section{Metodología}

La Facultad de Estudios a Distancia (Faedis) de la UMNG, entidad pública del orden nacional, en el presente estudio se traza la necesidad de corroborar el perfil de los alumnos de pregrado inscritos en el semestre 2018-II, y así poder contar con información que reconozca y enuncie aspectos esenciales como es la edad, el lugar geográfico donde residen, el estrato social, y la afiliación con la universidad. Al confirmar estas variables, se pude dar respuesta y posibles soluciones a problemas con el fin de demarcar las habilidades pedagógicas y metodológicas de los estudiantes.

El estudio se realizó mediante el paradigma interpretativo-comprensivo con enfoque cualitativo, y con metodología descriptiva. 
La elaboración y recolección de los datos obtenidos se realizó mediante una muestra probabilística aleatoria simple, en una herramienta (encuesta) que se ubicó en la página de la plataforma de la universidad, donde los estudiantes ingresan y trabajan sus asignaturas cursadas en la Facultad.

El número de estudiantes inscritos para el periodo 2018-II era de 2563 estudiantes activos en modalidad virtual, en los programas de la Facultad de Estudios a Distancia; la muestra que respondió la encuesta la conforman 363 educandos de manera aleatoria, que corresponde al $14,2 \%$ de la población.

\section{Resultados}

Con el instrumento que diligenciaron los alumnos de la Faedis (matriculados en modalidad virtual y a distancia) entre agosto y noviembre de 2018, se agruparon los datos de 363 , sobre una población de 2563 estudiantes, los cuales corresponden a pregrado $(92,7 \%)$ y posgrado $(7,3 \%)$.

Con la información suministrada en el periodo 2018-II, se realizó la comparación con los resultados del semestre 2015-II, la cual se presenta a continuación.

\section{Aspectos socioeconómicos}

Figura 1. Rango de edades de los estudiantes en los periodos 2015-II y 2018-II.

2015-II

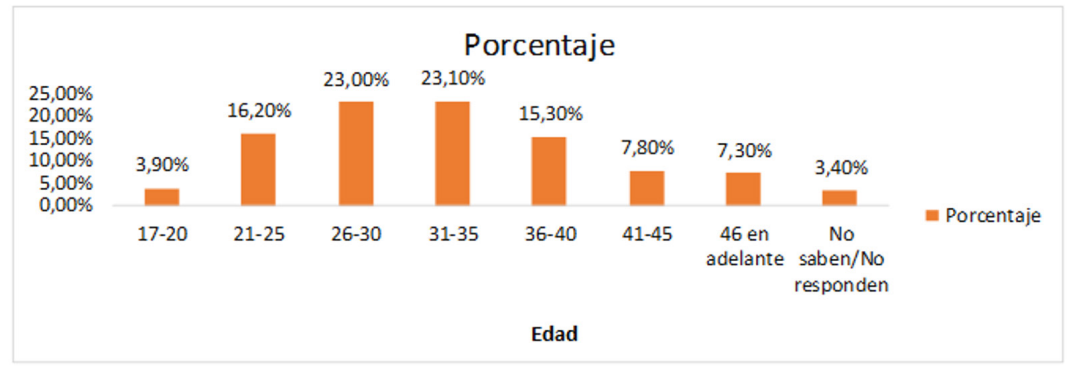




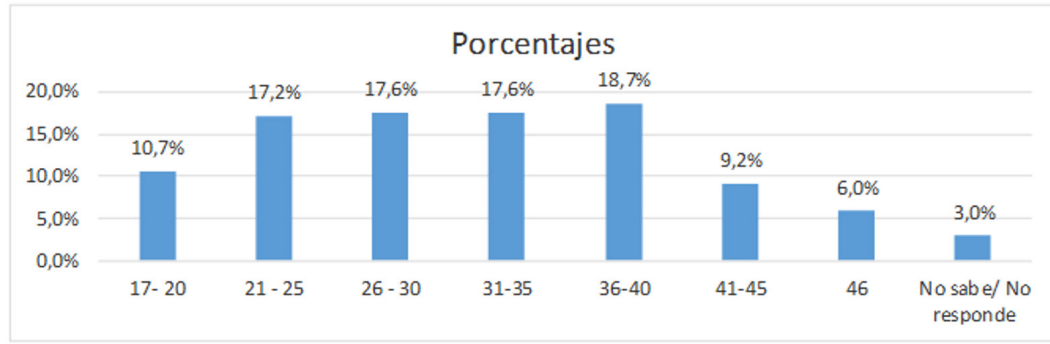

Fuente: elaboración propia.

Respecto a los grupos de edades de los estudiantes que desarrollaron el instrumento según lo establecido (figura 1), de esta comparación se puede determinar una similitud entre los dos grupos; sin embargo, llama la atención el incremento de estudiantes de edades más tempranas en esta modalidad, pues se incrementó en el rango 17 -20 años; este grupo aumentó en 6,8 \% puntos porcentuales, pasando de 3,9 \% (2015-II) a 10,7 \% (2018-II). Los demás rangos de edades en general mantienen similares sus porcentajes en ambos periodos.

Se reafirma con estos datos obtenidos que el rango de edades en el que más estudiantes se inscriben e ingresan, en esta modalidad virtual-a distancia, es el comprendido entre los 26 a 40 años, lo que corrobora que son una comunidad mayor de edad pero joven.

En lo concerniente al género de los estudiantes que respondieron el instrumento en los periodos 2015-II y 2018-II, los encuestados se mantienen con tendencias similares, dando como resultado que el $41,5 \%$ y el $43,1 \%$, respectivamente, son del sexo femenino; y un $58,3 \%$ y $56,9 \%$, respectivamente, son del sexo masculino.

En un estudio anterior, los profesores Silva y Espinel (2006) realizaron una encuesta a una población de la UMNG, cuyos resultados en modalidad presencial muestran que "dentro de las principales características, se evidencia que el $55,17 \%$ de la población es de género femenino y el restante $44,83 \%$, masculino" (p. 240). A su 
vez, Acuña (2014), en otro trabajo con los educandos de la UMNG en modalidad presencial, presenta que "con relación al género, la encuesta arrojó que la población femenina, con un 58,0\%, es la población predominante" (p. 156). Es un punto de análisis para tener presente que en más de una década el género masculino tiene mayor número de inscritos en la modalidad virtual-a distancia que en la modalidad presencial.

Figura 2. Estrato socioeconómico de los estudiantes en los periodos 2015-II y 2018-II.

\section{5-II}

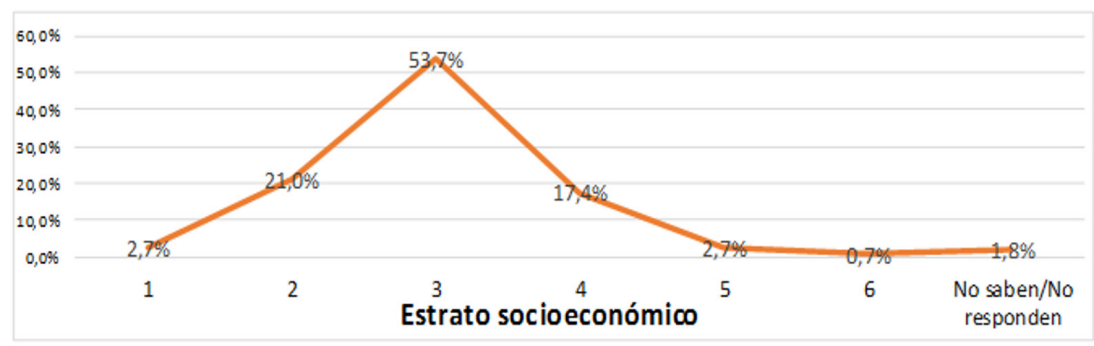

2018-II

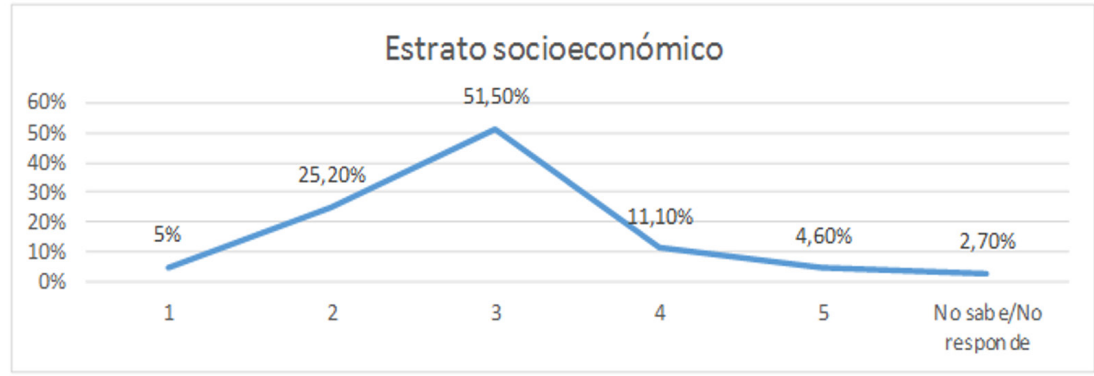

Fuente: elaboración propia.

Con relación al nivel socioeconómico predominante entre los alumnos que se inscriben a la modalidad virtual-distancia (figura 2 ), estos se encuentran principalmente en el estrato socioeconómico tres (en el que se ubica el $53,7 \%$ y el $51,5 \%$, respectivamente), al que le sigue el estrato dos ( $21 \%$ y $25,2 \%$, respectivamente).

Además, en comparación con la investigación anterior, para el periodo 2018-II el estrato 4 disminuyó 6,3 puntos porcentuales. 
Figura 3. Origen geográfico de los estudiantes en los periodos 2015-II y 2018-II.

\section{5-II}

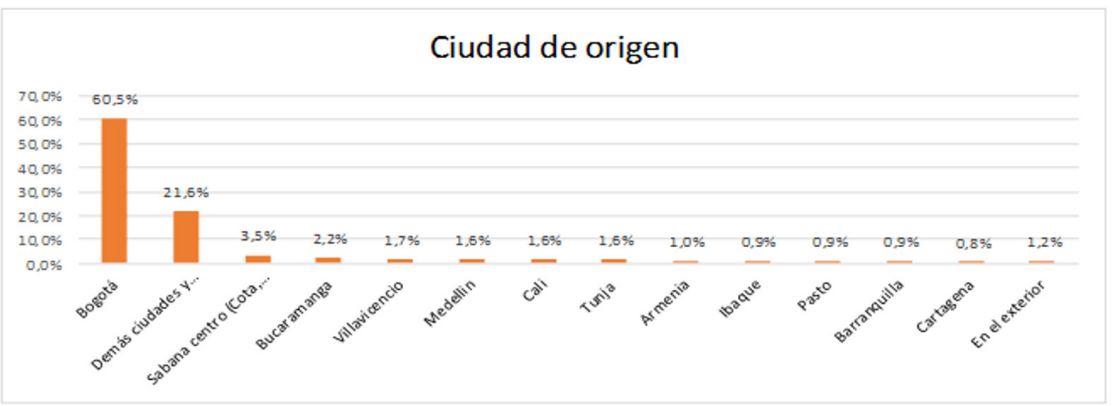

2018- II

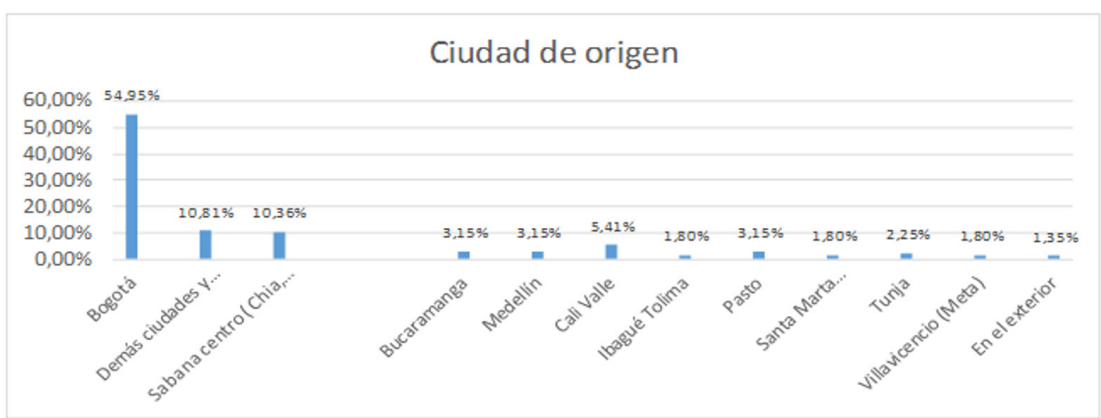

Fuente: elaboración propia.

Los estudiantes de la Faedis, con relación a su ubicación geográfica, se localizan principalmente en la ciudad de Bogotá $(60,5$ $\%$ en 2015-II, y 54,95 \% en 2018-II); a pesar de que la cifra tuvo un descenso de 5,55 puntos porcentuales, el resultado de los provenientes de la Sabana Centro tuvo un repunte importante de estudiantes, pasando de $3,5 \%$ a $10,36 \%$. 


\section{Aspecto económico de los estudiantes}

Figura 4. Situación laboral de los estudiantes en los periodos 2015-II y 2018-II.

2015-II

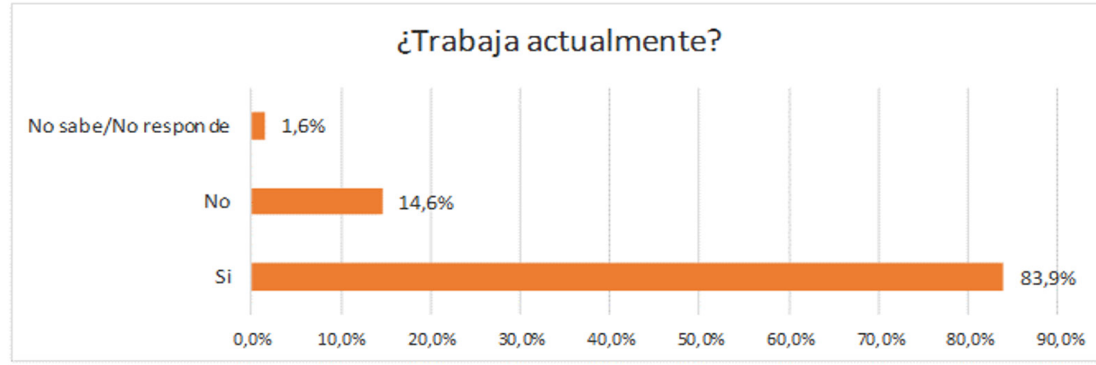

2018-II

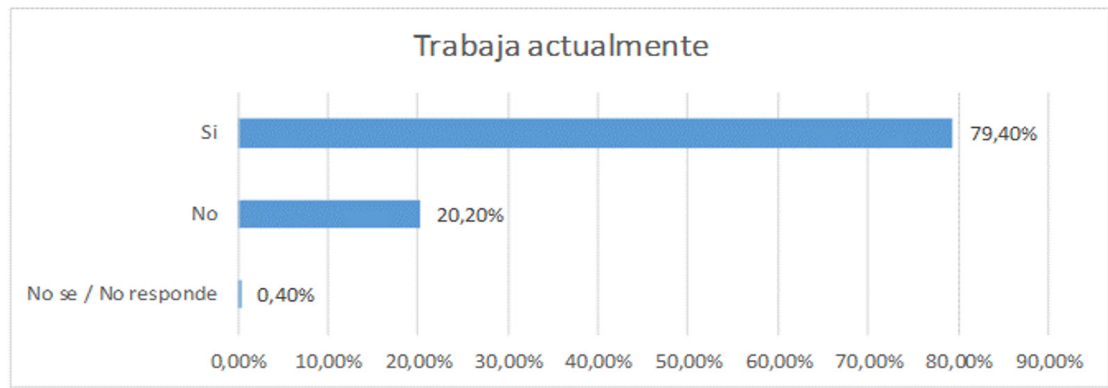

Fuente: elaboración propia.

En relación con el aspecto económico, los estudiantes matriculados en la Faedis presentan una fuerte dependencia de sus ingresos laborales para cancelar el costo en la matrícula y sostenimiento diario; así, en 2015-II los estudiantes respondieron que un $83,9 \%$ trabajan, situación similar al 79,40 \% del periodo 2018-II.

En referencia al estudio de 2015-II, Acuña, Padilla y Riaño (2016) afirman en sus resultados que:

[...] deben acudir a solvencias externas mediante: créditos bancarios $(84,1 \%)$, tarjetas de crédito $(8,4 \%)$, créditos 
educativos y becas en el exterior (Icetex) (1,4\%); y solo lo realizan con recursos propios (1,1\%); otros estudiantes acuden a su familia en un porcentaje de 0,9\%; mientras que $3,9 \%$ aún no tienen certeza de cómo financiar el próximo semestre. (p. 186)

Figura 5. Financiación del semestre en 2018-II.

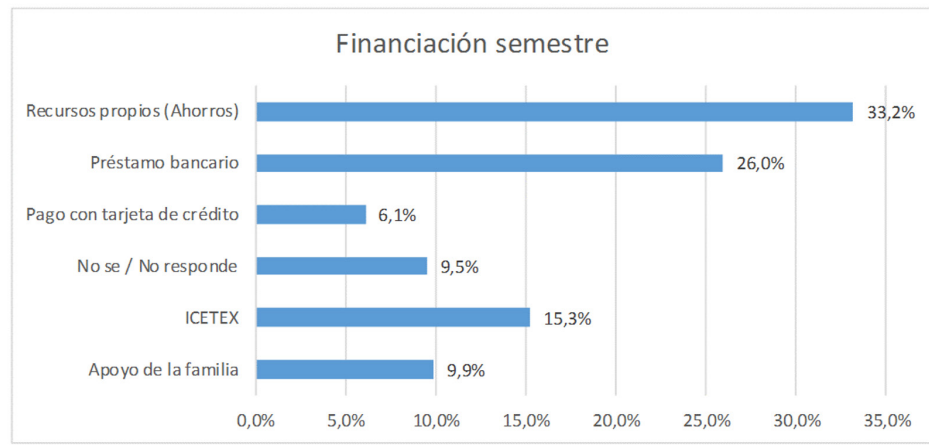

Fuente: elaboración propia.

Reiteran los encuestados en ambos estudios realizados que a pesar de tener un trabajo, cuando se matriculan a un programa en modalidad a distancia-virtual, para sufragar el costo de la matrícula deben acudir al ahorro propio, y a otro tipo de préstamos (figura 5), como lo son: crédito bancario ( $84,01 \%$ en 2015 -II y 26,0 $\%$ en $2018-$ II), uso de la tarjeta de crédito $(8,4 \%$ en 2015 -II y 6,1 $\%$ en 2018-II), y el crédito con el Icetex (1,4\% en 2015-II y 15,3\% en 2018-II).

Figura 6. Interés por los programas ofrecidos bajo la modalidad a distancia y virtual (periodos 2015-II y 2018-II).

\section{5-II}

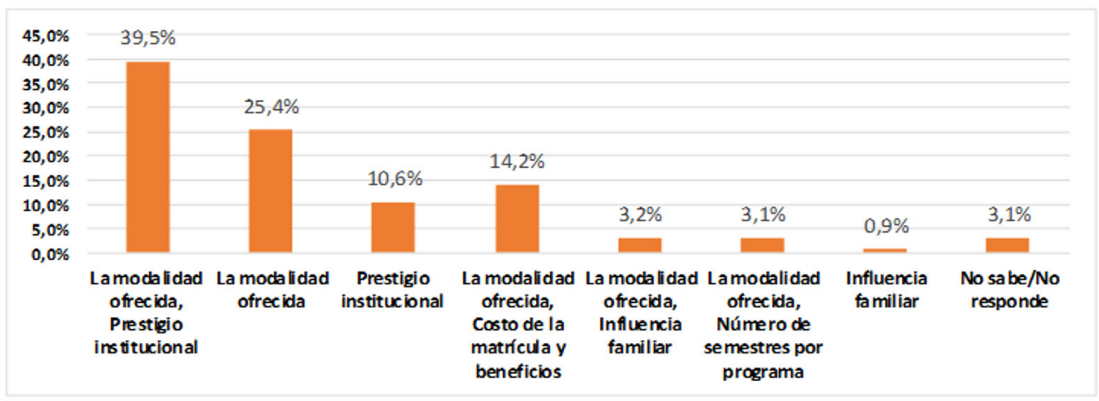




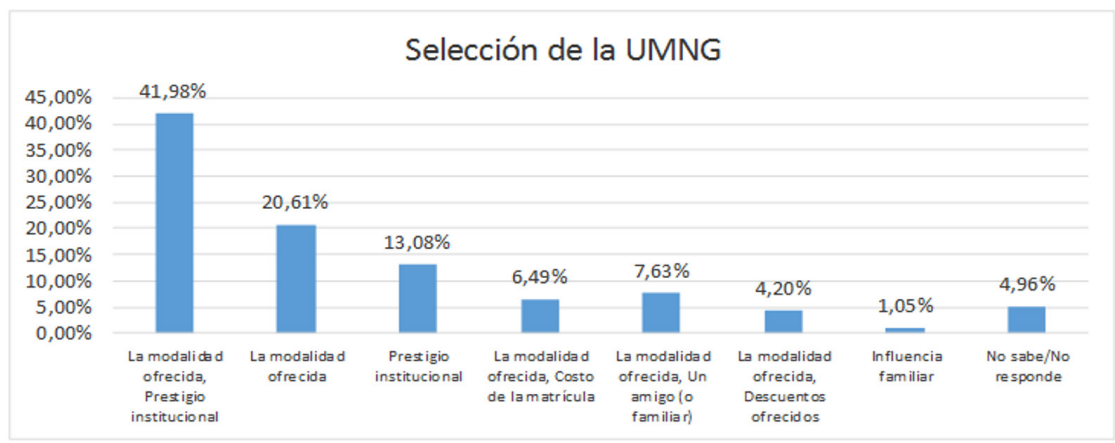

Fuente: elaboración propia.

En cuanto a la correspondencia entre la modalidad ofrecida y la selección de su IES, la mayoría de los alumnos que hacen parte de este estudio comparativo (2015-II y 2018-II) seleccionaron a la Faedis por la modalidad a distancia-virtual ofrecida y el prestigio institucional, razón que se mantiene como la más común e inclusive se incrementó en 2,48 puntos, pasando de 39,5\% a 41,98\% (figura 6); las demás variables mantienen su expectativa de una forma positiva.

Percepción que tienen los encuestados sobre cursar un posgrado a distancia

Figura 7. Posgrados solicitados por los estudiantes (periodos 2015-II y 2018-II).

\section{5-II}

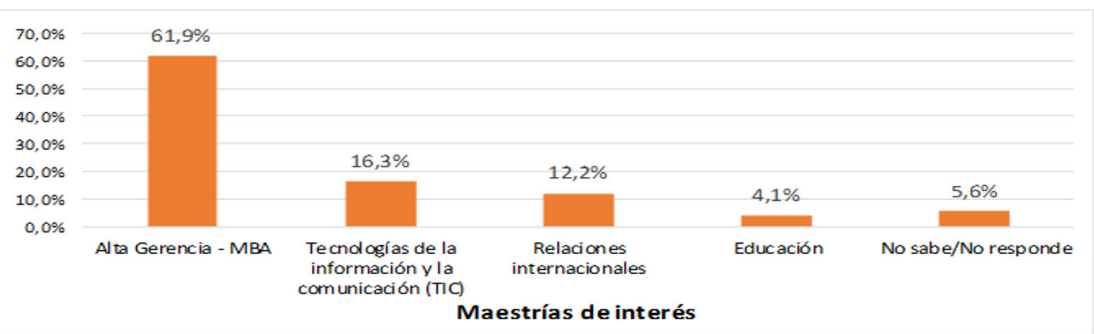


2018-II

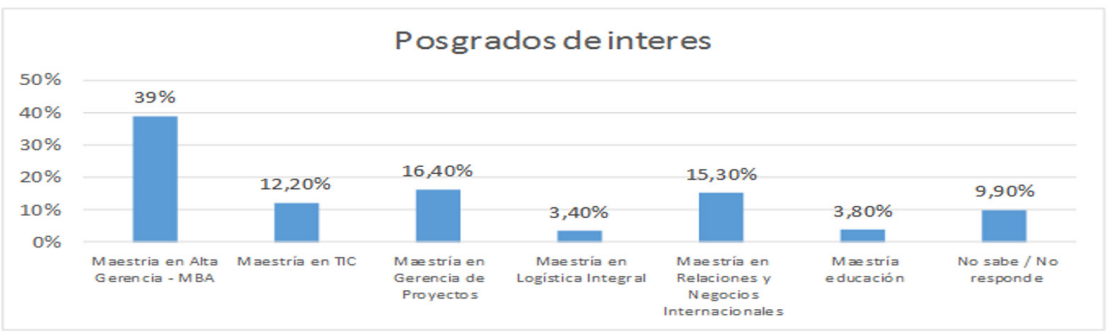

Fuente: elaboración propia.

Con relación a la pregunta sobre si desearían estudiar un posgrado a distancia con la Faedis, teniendo presente que en el corto plazo la UMNG va a ofertar mediante esta modalidad algunas especializaciones adicionales a la existente (Especialización en Alta Gerencia) y maestrías, haciendo una comparación de los resultados a esta misma pregunta en 2015-II y 2018-II, los interesados indicaron mantener el interés por algunos posgrados como son: Alta Gerencia (MBA), en un $61,9 \%$ y $39,0 \%$, respectivamente; Tecnología de la Información y la Comunicación, 16,3 \% y 12,20 \%; Relaciones internacionales, 12,2 \% y 15,30 \%; Educación, 4,10 \% y 3,80 \%; No sabe/no responde, también varios: $5,6 \%$ y $9,9 \%$.

Se observa un cambio significativo en la tendencia entre los años comparados (2015-II y 2018-II) en relación a la Maestría en Alta Gerencia (MBA), donde se evidencia una disminución en el interés. En 2018-II también se dio la opción de proponer posgrados de interés, entre los que Ilama la atención la maestría en Gerencia de Proyectos, con un $16,40 \%$. 


\section{Aspecto institucional}

Figura 8. Grado de afiliación con la UMNG (periodos 2015-II y 2018-II).

2015-II

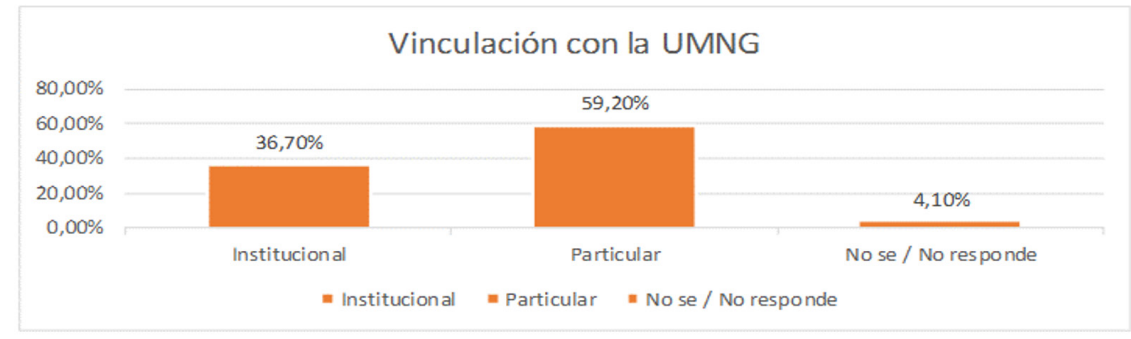

2018-II

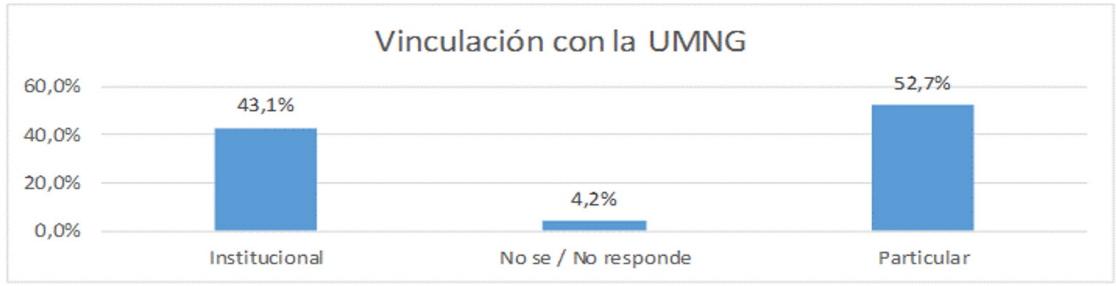

Fuente: elaboración propia.

Una de las preguntas buscaba comparar el vínculo actual (o grado de afinidad) de los estudiantes con la institución, sobre lo cual se presenta un cambio significativo en lo referente al incremento de vinculados institucionales, pasando del 36,7 \% (2015-II) al 43,1 $\%(2018-I I)$.

Al respecto, cabe recordar que el personal institucional está definido así según la Resolución 0799/2013:

1) Personal en servicio activo, con asignación de retiro o pensión: oficiales, suboficiales y soldados profesionales de las Fuerzas Militares; oficiales, suboficiales y agentes de la Policía Nacional. 2) Personal civil con vinculación actual como empleado público o trabajador oficial en el 
Ministerio de Defensa Nacional, las Fuerzas Militares, la Policía Nacional y las entidades adscritas y/o vinculadas al Ministerio de Defensa. 3) Personal administrativo de planta y docentes de tiempo completo y medio tiempo de la Universidad Militar Nueva Granada, con vínculo laboral vigente, después de haber cumplido el período de prueba. 4) Pensionados del Ministerio de Defensa Nacional y de la Universidad Militar Nueva Granada. 5) Los docentes de hora cátedra y personal administrativo temporal, con vínculo laboral vigente, y período de antigüedad no inferior a dos (2) años. 6) Los cónyuges del personal relacionado en los numerales 1) a 4). 7) Los hijos solteros menores de veinticinco (25) años del personal relacionado en los numerales 1) a 4). 8) Los egresados graduados de los programas de pregrado de la Universidad Militar Nueva Granada.

\section{Discusiones y conclusiones}

La modalidad a distancia-virtual es una realidad en Colombia y el mundo; su crecimiento casi exponencial hace que las IES continúen buscando mejores metodologías de aprendizaje, modernizando sus plataformas tecnológicas $y$, claro, ofertando programas innovadores para una población demandante de calidad en sus pregrados, posgrados y educación no formal; por lo anterior, es de suma importancia que las facultades que brindan este tipo de modalidad, y en especial la Faedis de la UMNG, continúen creciendo, especialmente en Bogotá (por tener su mayor número de estudiantes) pero también en todo el resto del país y del mundo. Para ello, se deben crear sedes, centros, unidades de atención (o como los deseen llamar) que ayuden a los estudiantes a estar más cerca de su universidad, con el fin de ofrecer la mejor educación, con los mejores medios tecnológicos y pedagógicos.

Se reitera que esta modalidad debe seguir creciendo, y más en las ciudades capitales, puesto que es en estas en donde se ubica el 
mayor número de interesados que requieren programas de educación superior en Colombia; claro, sin dejar de lado a las ciudades pequeñas y municipios del país, más ahora que esta modalidad aumenta en proporciones exponenciales, como lo manifiesta la Universidad EAN (2018) citando cifras del Ministerio de Educación Nacional:

En Colombia, aunque las cifras que arrojan las estadísticas siguen siendo tímidas, es un hecho que la educación virtual está tomando fuerza y se está abriendo camino a grandes pasos, sobre todo en la última década, en el 2010 había 12000 estudiantes en esta metodología, en el 2015 la cifra ascendió a 65000 y durante el 2017 llegó a 80 000. (p. 1)

Al presentar los resultados comparativos se evidencian y corroboran aspectos de los estudiantes encuestados que guardan relación con lo expresado por García Aretio (2006) en lo referente a que "ingresan alumnos que en conjunto forman grupos heterogéneos en cuanto a edad, experiencias o motivaciones, que tienen más ocupaciones y el estudio no es su única prioridad"(p. 122). Lo anterior guarda relación con la flexibilidad, apoyo y comprensión que debe tener el alumno que selecciona la modalidad a distanciavirtual, por ello lo importante que es el rol del tutor docente de conocer el perfil de estos aspirantes dentro de su proceso.

Otro tema que deben tener que manejar los estudiantes de toda modalidad es el tema de los apoyos y permisos de los empleadores para ingresar a carreras técnicas y profesionales; según el libro $L a$ educación en Colombia (Ministerio de Educación Nacional y OCDE, 2016), "la falta de compromiso de los empleadores en cuanto a la gestión y prestación de la educación superior implica que tanto esta como la formación ofrecida a menudo está desconectada del desarrollo local y las necesidades de empleo" (p. 302).

Revalidando lo anterior, se destaca la importancia de conocer al estudiante desde muchas variables y sus percepciones; en palabras de Zhang y Kenny (2010), "conocer su origen, características, 
ocupaciones y hasta rasgos culturales; esto debido a que en un curso en línea pueden inscribirse personas de diferentes partes del mundo con experiencias educativas y de vida muy diversas". Respecto a esto, a través de la comparación de los resultados alcanzados, tiene importancia presentar por lo menos cuatro aspectos que enaltecen al estudiante de la Faedis y que pueden ser similares en otros alumnos que eligen esta modalidad. El primero está relacionado con las expectativas de edad de iniciación de los educandos encuestados en la modalidad virtual-a distancia, en donde el grupo está entre los 26 a 45 años principalmente; sin embargo, llama la atención el incremento de la población del primer rango (17-20 años), que pasó de 3,9 \% (2015-II) a tener un porcentaje de 10,7 \% (2018-II), lo cual podrá apuntar a que este grupo se está interesando más por esta tipo de modalidad y aprendizaje. Como segundo aspecto, es notorio el aumento de estudiantes de la Sabana Centro (Cajicá, Chía, Zipaquirá, Cota, Cogua), pasando de 3,5\% (2015-II) a 10,81\% (2018-II), lo cual es importante pero predecible, por tener esta población la cercanía a la nueva sede de la Faedis en el Campus Cajicá. El tercero se refiere a que el estudiante de la Faedis es considerado un adulto joven, que trabaja (83,90 \% en $2015-I I$ y $79,40 \%$ en $2018-I I)$; sin embargo, acuden al crédito bancario y a su familia para costear su matrícula. Y cuarto, en relación con el grado de afinidad con la UMNG la población neogranadina de la Faedis se están matriculando más en la modalidad virtual (es decir, toman el beneficio institucional, según Resolución 0799 de 2013, por el cual tienen derecho al descuento), toda vez que pasó de tener un $36,7 \%$ (2015-II) a un 43,1\% (2018-II).

En cuanto al interés específico que les llevó a optar por la UMNG y la Faedis, los estudiantes reconocieron que fue por la modalidad a distancia-virtual y el prestigio institucional $(75,5 \%$ en 2015 -II, en relación con 75,67 \% en 2018 -II), lo cual evidencia que el nombre de la Universidad y la facultad mantiene una excelente imagen institucional en esta modalidad para los estudiantes matriculados y futuros aspirantes que deseen ingresar a los pregrados virtuales de la UMNG. 
En lo concerniente a la pregunta sobre "posibles maestrías en el corto plazo", se encontró que los alumnos demandan que en el corto plazo la Faedis brinde posgrados (maestrías y especializaciones) en esta modalidad, principalmente en Maestría en Alta Gerencia (MBA) (61,9 \% en 2015-II); sin embargo, para esta en particular bajaon quienes tienen intención de cursarla, dado que para este segundo periodo evaluado obtuvo un $39 \%$ (2018-II). Lo anterior podría indicar lo dinámica y cambiante que es la intención de los estudiante interesados; frente a lo que la facultad debe estar preparada (con estudios de mercado focales a las poblaciones interesadas).

En relación con el estudio (2015-II y 2018-II), se mantiene una tendencia geográfica en los estudiantes que cursan sus pregrados en la Faedis en cuanto a su lugar de residencia, ya que principalmente se ubican en Bogotá $(60,5 \%$, 2015-II, y 54,95\%, 2018-II), a pesar que se ha aumentado el porcentaje de alumnos de la Sabana Centro en 10,81\%; por esto se podrían presentar problemas para la toma de las monitorias los sábados (día que más se programa); según Acuña, Padilla y Riaño (2016), "resulta de suma importancia desarrollar un estudio para observar la viabilidad de realizar algunas monitorías en la sede calle 100" (p. 191) para los estudiantes, por la distancia entre Bogotá y Cajicá .

Finalmente, a modo de reflexión nos señala la doctora Abril (2018):

El modelo de entorno virtual mediado por las tecnologías requiere en el estudiante la construcción y reconstrucción de sus capacidades básicas cognitivas, respondiendo a una experticia específica de una herramienta, así como sus estrategias de aprendizaje, factores afectivos, motivacionales, necesidades de aprendizaje, metas y expectativas, que le admitan gestionar su propio proceso de formación logrando un aprendizaje, incorporando así en su diario vivir redes de conocimiento, y permitiendo de esta forma ejercer libertad y apropiación de ciencias, fortaleciendo el statu quo de su aprendizaje. (p. 55) 
A modo de conclusión, es innegable, con todo lo anteriormente expuesto, que es imperioso y necesario conocer al detalle el perfil de los alumnos que toman la modalidad a distancia-virtual, no solo desde el momento de su admisión, sino en todo su proceso pedagógico; por ello, entre más aprendamos de ellos, menor será la deserción en esta modalidad.

\section{Referencias bibliográficas}

Acuña, H. (2014). El perfil del estudiante de pregrado de la facultad de ciencias económicas y su percepción sobre la Universidad Militar Nueva Granada (UMNG). Revista Educación y Desarrollo Social, 8(2), 146-167 DOI: https://doi.org/10.18359/reds.301

Acuña, H., Padilla, E. y Riaño, F. (2016). El perfil del estudiante de pregrado de la Facultad de Estudios a Distancia de la Universidad Militar Nueva Granada. Revista Interamericana de Educación, Pedagogía y Estudios Culturales, 9(2), 179-195. DOI: https://doi.org/10.15332/ s1657-107X.2016.0002.10

Abril, M. (2018). Motivación del aprendizaje en línea. Panorama, 12(22), 43-56. DOI: http://dx.doi.org/10.15765/pnrm.v12i22

Congreso de Colombia. (28 de diciembre de 1992). Ley 30 de 1992. Por el cual se organiza el servicio público de la educación superior (art. 53). Recuperado de http://www.mineducacion.gov.co/1621/articles-85860_ archivo_pdf.pdf

García Aretio, L. (2006). Perfiles Educativos, vol. XXXIV, núm. 136, 2012, pp. 118-136 Instituto de Investigaciones sobre la Universidad y la Educación Distrito Federal, México Recuperado de https://www.redalyc. org/pdf/132/13223068008.pdf

Ministerio de Educación Nacional y OCDE. (2016). La educación en Colombia: revisión de políticas nacionales de educación. Recuperado 
de https://www.mineducacion.gov.co/1759/articles-356787_recurso_1. pdf

Resolución 0799 de 2013. C. Institucionalidad: Recuperado de https:// www.umng.edu.co/transparencia/informacion-de-interes/preguntas-yrespuestas-frecuentes/division-financiera

Silva, A. y Sarmiento, J. (2006). Estudiantes de Economía de la UMNG, ¿cómo son? Revista Facultad de Ciencias Económicas: Investigación y Reflexión, XIV (1), 238-257. Recuperado de https://www.researchgate. net/publication/237042068_Estudiantes_de_economia_de_la_UMNG_ como_son\#fullTextFileContent

Universidad EAN. (2018). La educación virtual se abre camino en Colombia. Recuperado de https://universidadean.edu.co/noticias/laeducacion-virtual-se-abre-camino-en-colombia

Zhang, Z. y Kenny R. (2010) Learning in an Online Distance Education Course: Experiences of Three International Students. International Review of Research in Open and Distance Learning, 11(1), 18-36. Recuperado de http://www.scielo.org.mx/scielo.php?script=sci_arttext \&pid=S0185-26982012000200008 